\title{
Research on Optimization of Formula SAE Truss-Frame
}

\author{
Yu Wang ${ }^{1}$, Jianwei Liu ${ }^{2}$, Yuhan $\mathrm{Li}^{1}$ and Nianjiong Yang ${ }^{3}$ \\ ${ }^{1}$ Guilin University of Electronic Technology, School of Mechanical and Electrical Engineering, 541000 Guilin, China \\ ${ }^{2}$ Guilin University of Electronic Technology, Department of Practice Teaching, 541000 Guilin, China \\ ${ }^{3}$ Guangxi University of Science and Technology, Guangxi key laboratory of Automobile Components and Vehicle technology, \\ 545006 Liuzhou, China
}

\begin{abstract}
The frame, as one of the basic components of a car, plays an important role of connecting components and withstanding various loads. The truss-frame is the commonest form of Formula SAE car in that it has many advantages, such as low cost, simple manufacture, high rigidity and high strength. The development of frame is reviewed. First, the key performance indicators and the mechanical state of the frame under different conditions are discussed. Second, a variety of optimization methods used in the design of frame are described. Finally, the test method of frame are introduced, with the experimental data and the finite element analysis of results being compared. Through analyzing and summarizing the development of the design, optimization and testing technology of the Formula SAE truss-frame, forecasting the future trends, the great theoretical reference is provided for the design and research of the follow-up frame.
\end{abstract}

\section{Introduction}

Formula SAE (FSAE) was originally initiated by the American Society of Automotive Engineers for college students in a formula car race. The FSAE event was organized by China in 2010, with 20 college teams participating. In 2015 , the number of college teams had reached 67.

The frame of Formula Student China carries the engine, body and various ancillary facilities. The structure strength of frame greatly affects the car's safety, power, comfort, handling stability and other performance indicators [1]. To ensure the normal functioning of the frame, the FSAE car frame must have sufficient rigidity, strength. And the ergonomic requirements are taken into account. The light-weight design of frame is an important goal in that the lighter frame will help improve acceleration performance and fuel efficiency. Thus, the high strength, high stiffness and light weight of the frame to improve race performance has a significant impact by the college team's attention.

There are two common frame types, one is the monocoque structure, the other is the truss structure. The monocoque is a shell frame made of carbon fiber, which has light weight, high strength and so on. However, the expensive price, complex production process and other issues limit its use in college teams. Truss-frame is inexpensive and simply manufactured, having now been adopted by most college teams. Therefore, this paper details the truss-frame's mechanical properties, optimization and testing methods.

\section{Mechanical analysis of frame}

Mechanics analysis is the basis of structural design. According to the load condition of the frame, an accurate equivalent mechanical model is proposed, which is helpful to understand the mechanism of the force and deformation of the frame. The favorable theoretical foundation is provided for optimization of the frame.

\subsection{Working conditions of frame}

From bending conditions, reversing the condition, a combination of bending and torsion conditions, lateral acceleration conditions, longitudinal acceleration condition and a combination of horizontal and vertical acceleration conditions, the FSAE car frame is analyzed.

The bending condition is the condition of bending deformation of the frame, which is caused by the gravity of the driver and other parts of the vehicle. The torsional condition is the condition of the torsional deformation of the vehicle frame, which is caused by the uneven stress of the four tires. A single torsion condition is not exist. Because the frame torsion deformation, it is bound to bear the gravity of the driver and the parts. Therefore, it could only be carried out on the frame of the other five kinds of working conditions, with ergonomics being considered. In the course of driving, the main forms of motion include uniform speed, acceleration, braking and turning. According to these forms, the common condition of the vehicle frame can be divided into three kinds, that is, the bending condition, the accelerating condition and 
the turning condition. Dynamic load coefficient was added in the analysis of the strength of the frame under each condition. By this method, accurate results could be obtained, with the efficiency of the analysis being improved.

\subsection{Kinetics analysis}

The static analysis can not meet the design requirements of the frame. Dynamic analysis and optimization of dynamic performance are of great significance to improve the performance of the frame.

Resonance can be caused, reducing the comfort of the vehicle and increasing the load of the frame when the natural frequency of the frame is close to the excitation frequency of road surface or the engine. The natural frequencies and vibration modes of the frame were obtained by the modal analysis. The frame structure was optimized, so that the natural frequency of the frame to avoided the road and engine vibration frequency. To further analyze the performance of the frame, the frequency of the engine and suspension assembly on the frame were analyzed. This frequency needed to avoid tire rotation frequency of the normal speed.

The vehicle collision test is an important way to study the safety of vehicles, but most of the college team does not have the test conditions. Therefore, it is an ideal way to study the safety of the vehicle by using the computer simulation of vehicle collision. The crash simulation of $40 \%$ offset frontal was carried out to study the change of acceleration and energy during the collision and the deformation and damage of frame structure after impact.

\subsection{Torsional stiffness}

Torsional stiffness and weight are the two most important quantifiable aspects to the frame of any race car. Thus, the first aim of this project is to design a frame of which the trade-off between high torsional stiffness and low weight is balanced to achieve high vehicle performance across the various competition events [1].

As a method for analyzing and balancing the frame, the frame was divided into three main section, the front bulkhead, the driver compartment and the rear section. A schematic diagram is shown in Fig. 1. The torsional stiffness of each part was analyzed separately. The total stiffness of the frame can be expressed by equation (1). The $\mathrm{K}$ is the total stiffness of the frame. The $\mathrm{kf}$ is the stiffness of the front bulkhead. The kd is the stiffness of the cockpit. The $\mathrm{kr}$ is the stiffness of engine compartment.

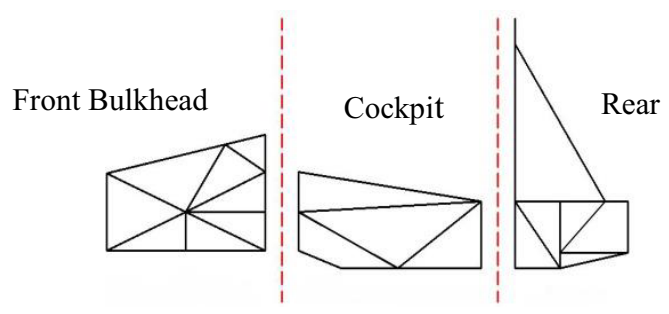

Figure 1. Divide frame figure

$$
\frac{1}{K}=\frac{1}{k_{\mathrm{f}}}+\frac{1}{k_{\mathrm{d}}}+\frac{1}{k_{\mathrm{r}}}
$$

The equivalent mechanical model was established, as shown in Fig. 2. Further analysis of the various parts of the frame, it could be found that the cockpit is one of the important factors affect the level of the vehicle stiffness. By increasing the cockpit section, the torsional rigidity of the frame could be improved effectively. The smaller front is helpful to reduce the torque. Engine parts such as stiffness is bigger, reasonable installation can effectively increase the nacelle stiffness. So, the reasonable frame structure should be compact front, wide of the cockpit and compact engine compartment.

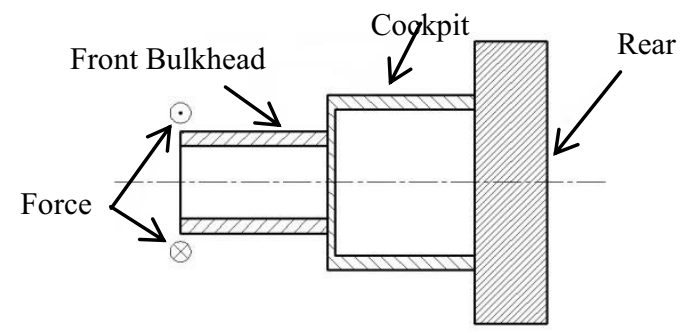

Figure 2. The equivalent mechanical model

Analysis the stress and the deformation law of frame is the basis of optimized frame. The most basic requirements is that frame can work in a variety of conditions. Torsional stiffness of the frame is an important performance index. High torsional stiffness and light weight are the goal of optimizing the frame. According to the research of Jacob I. Salter, William B. Riley and Albert R. George et al. [2], [3], a small cabin could effectively reduce the torsional moment of the frame. The torsional performance of frame could be increased by the large cockpit, with engine and other major components increasing the stiffness of the rear frame stiffness. The research of W. Billygolfer and K. Kcarney et al. [4] shows that the engine compartment can be omitted, in order to further reduce the weight of the frame and improve the performance of the frame. This structure is shown in Fig. 3. This space layout provides a good reference for the frame structure design.

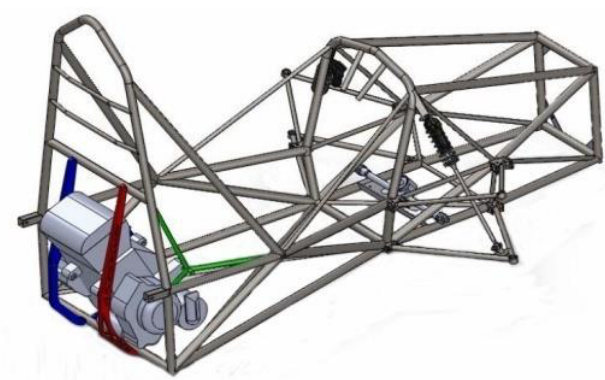

Figure 3. The structure of frame

\section{Optimization method}

The truss-frame is made up of steel tubes. The spatial position, thickness and many other factors of the steel tubes have great influence on the strength, rigidity and 
weight of the frame. Optimization algorithm has become an important method to optimize the frame.

\subsection{Topology optimization}

Topology optimization is a mathematical method to optimize the distribution of materials in a given region, according to the given load condition, constraint condition and performance index. This method provides designers with an ideal structure for reference.

Finite element model was established by using shell element. The topology optimization was carried out under six conditions. Considering the force of the frame, the structure of the final frame was designed. This method can provide a variety of references for the conceptual design of the frame, with allowing a more rational arrangement of steel pipe and reducing the quality of the frame.

\subsection{Multi-objective optimization}

The truss frame is made up of many different sizes of steel tubes, with the parameters of each steel tube affecting the performance of the frame. It is difficult to find the appropriate value in many of the design parameters. Therefore, the multi-objective optimization has become one of the most widely used method for frame optimization.

The section size of steel tube was set as the optimization variable. The stress and deformation were set as constraint conditions. The stiffness, vibration frequency and quality of frame were set as the objective function. The sensitivity analysis of frame was carried out, with the correlation between size of steel tube and frame stiffness, vibration frequency and mass being obtained. The appropriate size parameters were obtained by response surface analysis of some steel tubes.

There are two main difficulties in the design of the truss frame. One is the rational design of space structure. The other is the choice of the size of the pipe. The new spatial layout and optimum material distribution program can be provided by the topology optimization. Multiobjective optimization can find the suitable values in a large number of design parameters. It provides an effective way to optimize the structure size. According to the research of Fu Yuanfang and Wang Shuting [5], [6], using the optimization algorithm is an effective method to obtain the excellent performance of the frame.

\section{Test method of the frame}

Using the finite element software to analyze the frame is an important method to get the performance parameters of the frame. It is also one of the most popular methods to study the frame. However, the results obtained from the finite element software have a certain error with the actual performance. Therefore, testing the physical frame is of great significance in that it can provide more accurate data.

The common torsional stiffness measurement method is measured by the sensor. Some pressure sensors are installed on the four test points of front and rear suspensions. Two test points of rear suspension are fixed on the test table, with a pair of forced displacement being applied on two test points of the front suspension. By measuring the force and torsion angle, the actual torsional rigidity of the frame is calculated. A simple measurement method is that two rear suspension test points and right front suspension test points were fixed. The load would be applied by attaching a steel rod through the front bulkhead of the chassis, with the second rear rod being used to counter acts the moments created by the first and thus stabilized the rig. A horizontal bar was set up in front of the frame. Weight would then be added to both rods, with the angle between the center line of the front bulkhead and the horizontal bar being measured. According to this angle, the torsional stiffness was calculated. The schematic diagram of the experiment is shown in Fig. 4:

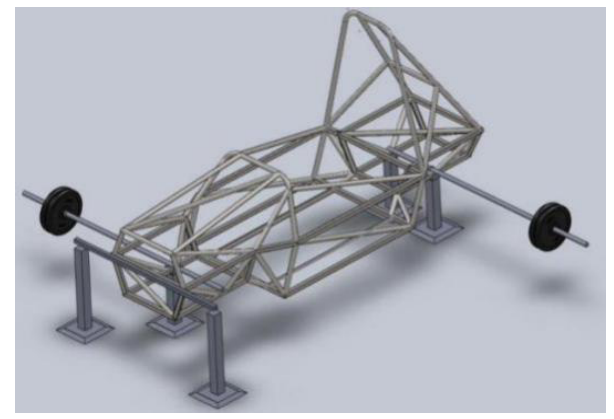

Figure 4. The measurement device of torsional stiffness

The angle of wheel is an important parameter to ensure the smooth running of the vehicle. When the frame occur deformation, the relative position of the fixed point of the suspension is changed, with the angle of wheel being changing. The ride comfort of vehicle also deteriorates. A new test method is that the suspension is mounted on the frame, with an aluminum plate being installed in the column. The sensor was mounted on the aluminum plate. Restraint and load was applied on the aluminum plate. The stiffness of frame was measured. At the same time, the change law of the tire angle was obtained. The comprehensive data reference of frame optimization was provided. The experimental apparatus is shown in Fig. 5:

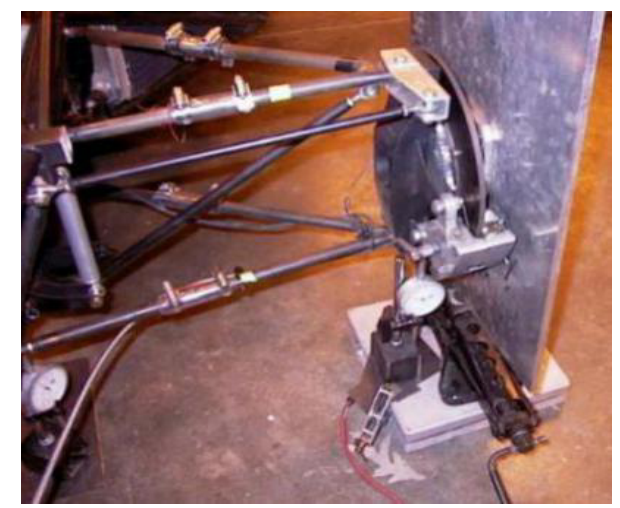

Figure 5. The experimental apparatus

Due to the influence of positioning error and welding distortion, the actual stress, strain and the design value of the frame have certain error. The measurement method 
proposed by Jacob I. Salter and Thompson et al. [3], [7] could accurately measure the stiffness and strength of the frame. William B. Riley and Albert R. George et al. [2] put forward a new method. This method of applying the load through the suspension to measure not only can measure the rigidity of the frame, but also can measure the variation of the working angle of the tire, which provides accurate data for the analysis of vehicle performance. The accurate data for the strength analysis of the frame is provided.

\section{The development trend}

After reasonable design, the truss-frame can be used to meet the requirements. Thanks to its simple and easy implementation, truss-frame has become the mainstream of the frame form the team of University. Through the analysis, several development of the truss-frame are listed. First, the core goal is lightweight. Second, the front bulkhead is more compact, more spacious cockpit. Third, optimization method is applied more widely. Last, the test equipment of Frame will be improved.

\section{Conclusions}

With the promotion of the FSAE tournament, the research of the truss frame has been developed, but there are still a number of deficiencies. The vehicle collision test is an important part of vehicle safety research. Due to limited experimental conditions, the University team is difficult to carry out relevant tests. Using the computer to simulate the vehicle collision is the best way to study this kind of problem, but there is little research on the FSAE racing crash in China. In view of the test equipment of the FSAE truss-frame is less, design of the test equipment for the University team to use is an important problem to be solved.

The competition of Formula Student China start time is late, compared with the developed countries, so the technology and management of China are backward. At present, the design and manufacturing technology of Chinese automotive are weak. Through the competition of FSAE, the students' engineering practice ability is tempered. In 2010, the first FSAE competition was held. After six years of development, the number of college teams is more, with the car being getting better and better.
The frame of Formula Student China carries the engine, body and various ancillary facilities. The frame designers all hope that frame has high strength and light weight. Therefore, it is significantly important for the development of car to research frame design and optimization.

\section{Acknowledgment}

The authors are thankful to the Guangxi Natural Science Foundation (Grant no: 2013GXNSFBA019245), Science and technology research project of Guangxi University (Grant no: KY2015YB095), the Opening Project of Guangxi Key Laboratory of Automobile Components and Vehicle Technology, Guangxi University of Science and Technology (No. 2014KFMS05), and the graduate student scientific research innovation projects of Guilin University of electronic technology (Grant no: YJCXS201504)

\section{References}

1. X. Zhang, B. Gao, Y. Wang, The finite element analysis of FSC car under different conditions, Manufacturing automation, 37, 10, 44-46 (2015)

2. W. Riley, A. George, Design, Analysis and Testing of a Formula SAE car chassis, SAE technical paper series, 2002-01-3300 (2002)

3. J. Salter, Design, Analysis and Manufacture of 2011 REV FSAE Vehicle Chassis, The University of Western Australia (2011)

4. W. Billygolfer, K. Kcarney, J. Jleith, A. Antonk, Design and Optimization of a Formula SAE race car, Worcester Polytechnic Institute (2012)

5. S. Wang, X. Liu, Y. Wu, G. Jin, Lightweight and fatigue life estimation of frame based on sensitivity analysis, China Mechanical Engineering, 22, 16, 2001-2006 (2011)

6. Y. Fu, D. Jin, Y. Qiao, Optimum design method for frame structure of mini electric vehicle, Journal of Mechanical Engineering, 45, 9, 210-213 (2009)

7. L.L. Thompson, J.K. Lampert, and E.H. Law, Design of a Twist Fixture to Measure Torsional the Torsional Stiffness of a Cup Chassis, SAE Paper, 983054 